\title{
Importancia de la determinación de variantes en el número de copias en neonatos con aneuploidías autosómicas
}

Hugo Abarca ${ }^{1,2,3}$, Milana Trubnykova ${ }^{1}$, Félix Chavesta ${ }^{1}$, Marco Ordóñez ${ }^{4}$, Evelina Rondón ${ }^{4}$

${ }^{1}$ Servicio de Genética y EIM, Instituto Nacional de Salud del Niño-Breña, Lima, Perú

${ }^{2}$ Carrera Profesional de Medicina Humana, Universidad Científica del Sur, Lima, Perú

${ }^{3}$ Facultad de Medicina Humana, Universidad Ricardo Palma, Lima, Perú

${ }^{4}$ Escuela de Medicina Humana, Universidad Nacional de San Antonio Abad de Cusco, Cusco, Perú

Introducción. Las aneuploidías son trastornos genéticos frecuentes en la práctica clínica; sin embargo, se conoce poco sobre las otras variantes genéticas que modifican el fenotipo final.

Objetivo. Determinar las variantes en el número de copias y las regiones con pérdida de heterocigosidad autosómica mayor de $0,5 \%$ o de regiones mayores de $10 \mathrm{Mb}$ en neonatos con aneuploidías autosómicas.

Materiales y métodos. Se hizo el análisis cromosómico por micromatrices a los neonatos con aneuploidías autosómicas $(n=7)$, trisomía $21(n=5)$ y trisomía $18(n=2)$ evaluados en los hospitales Antonio Lorena y Regional de Cusco, Perú, en el 2018.

Resultados. En dos neonatos se encontraron variantes en el número de copias, patogénicas o probablemente patogénicas, en regiones diferentes al cromosoma 21 o al 18. Además, se observaron dos variantes del número de copias con más de $500 \mathrm{kpb}$ de patogenia desconocida.

Conclusiones. Si bien el número de pacientes era muy reducido, es importante resaltar que se encontraron otras variantes en el número de copias que se han descrito asociadas con trastornos del neurodesarrollo, varias anomalías congénitas, hipoacusia y talla baja o alta, entre otras, lo que probablemente influye negativamente en el fenotipo de este grupo de pacientes.

Palabras clave: aneuploidía; variaciones en el número de copia de ADN; recién nacido; trastorno del neurodesarrollo; sordera.

Importance of determining variations in the number of copies in newborns with autosomal aneuploidies

Introduction: Aneuploidies are frequent genetic disorders in clinical practice. However, little is known about other genetic variants that may influence the final phenotype.

Recibido: 09/01/2020

Aceptado: $17 / 12 / 2020$

Publicado: 14/01/2021

Citación:

Abarca $\mathrm{H}$, Trubnykova M, Chavesta F, Ordóñez

M, Rondón E. Importancia de la determinación de variantes en el número de copias en neonatos con aneuploidías autosómicas. Biomédica. 2021;41:282-92. https://doi.org/10.7705/biomedica.5354

\section{Correspondencia:}

Hugo Hernán Abarca, Servicio de Genética y EIM, Instituto Nacional de Salud del Niño, Avenida Brasil 600, CP Lima 05, Lima, Perú

Teléfono: (51 97) 9301132

habarca@insn.gob.pe

Contribución de los autores:

Todos los autores participaron en el diseño del estudio, la recolección de datos o pacientes, el análisis de resultados y en la redacción del manuscrito.

Financiación:

El estudio fue financiado por la Universidad Naciona de San Antonio Abad de Cusco.

Conflicto de intereses:

Ninguno declarado por los autores
Objective: To determine the variations in the number of copies and regions with

homozygosity greater than $0.5 \%$ or larger than $10 \mathrm{Mb}$ in newborns with autosomal aneuploidies.

Materials and methods: We performed a chromosomal microarray analysis on newborns with autosomal aneuploidies $(n=7)$, trisomy $21(n=5)$, and trisomy $18(n=2)$ evaluated at the Hospital Antonio Lorena and Hospital Regional of Cusco, Perú, during 2018.

Results: We found pathogenic and probably pathogenic variants in the number of copies in other genomic regions different to chromosomes 21 or 18 in two neonates. Additionally, we found two variants bigger than $500 \mathrm{kpb}$ of unknown pathogenicity.

Conclusions: Although the number of analyzed individuals was small, it is important to highlight that we found other variants in the number of copies that have been described in association with neurodevelopmental disorders, congenital anomalies, deafness, and short/ tall stature, among others, in almost half of them, which will probably impact the phenotype negatively in patients with aneuploidies.

Keywords: Aneuploidy; DNA copy number variations; infant, newborn; neurodevelopmental disorders; deafness.

Las aneuploidías autosómicas más frecuentes en recién nacidos son la trisomía 21 (síndrome de Down), la trisomía 18 (síndrome de Edwards) y la trisomía 13 (síndrome de Patau) (1). En Estados Unidos, se estima que uno de cada 732 recién nacidos vivos presenta el síndrome de Down, en tanto que la incidencia reportada del síndrome de Edwards es de uno de cada 4.857 a 10.000 y, la del síndrome de Patau, de 1,4 de cada 10.000 (2-4). 
El análisis cromosómico por micromatrices permite detectar las variaciones en el número de copias (Copy Number Variation, CNV), las cuales se definen como segmentos de ADN iguales o mayores de $1 \mathrm{kpb}$; además, esta técnica permite describir regiones de homocigosidad (Regions of Homozygosity, $\mathrm{ROH}$ ), cuyo índice se utiliza para evaluar disomías uniparentales y consanguinidad parental no declarada (5).

Las variaciones en el número de copias son frecuentes en el genoma humano e influyen en la variación fenotípica interindividual (6); pueden ser de pérdida o de ganancia y provocan un fenotipo determinado según el número de genes comprometidos o si son genes cuyo efecto depende del número de copias, la modificación transcripcional o la fusión génica $(7,8)$.

Dichas variaciones resultan en fenotipos con discapacidad intelectual, retraso del desarrollo psicomotor, epilepsia, trastorno del espectro autista, esquizofrenia y anomalías congénitas $(9,10)$. Por ejemplo, las variaciones de ganancia de los genes BCAP31 y SLC6A8 se asocian con hipoacusia no sindrómica $(11,12)$ y, las de los genes SHOX y PAK2, con trastornos del espectro autista y del neurodesarrollo (13-21). También, se ha descrito la relación de las variaciones en el número de copias y las anomalías congénitas, como cuando hay compromiso del gen TTC28 y se producen anomalías congénitas del tubo digestivo (22). Debe señalarse que algunas de estas variaciones se han descrito solamente a partir de bases de datos, como la DECIPHER (Database of Chromosomal Imbalance, Phenotype in Humans using Ensemble Resources) (23), y que las manifestaciones clínicas son pleiotrópicas, como la variación de ganancia en el cromosoma 16 (p12.2), la cual se ha relacionado con trombocitopenia, trastornos del neurodesarrollo y riñones en herradura $(23,24)$.

También, se ha reportado que algunas variaciones frecuentes en el número de copias están asociadas con enfermedades comunes, como infección por HIV, glomerulonefritis, lupus eritematoso sistémico, enfermedad de Crohn, psoriasis, osteoporosis, neuroblastoma, talla baja, fisura labiopalatina y obesidad; en este mismo sentido, algunas variaciones raras se han relacionado con la enfermedad de Parkinson, la enfermedad de Alzheimer y el trastorno bipolar (6).

En el síndrome de Down, se observan características clínicas diversas, por ejemplo, discapacidad intelectual, cardiopatías congénitas (40\%), defectos gastrointestinales ( 8\%), leucemia linfoblástica ( 20\%) o trastorno mieloproliferativo transitorio neonatal $(\sim 10 \%)$.

Se ha descrito que las variaciones frecuentes en el número de copias del mismo cromosoma 21 pueden determinar, por ejemplo, un mayor riesgo de enfermedad de Alzheimer, leucemia megacarioblástica aguda, hipoacusia o defectos congénitos (6). Es así que, en pacientes con trisomía 21, se ha observado que las variaciones de pérdida del cromosoma 21 (por ejemplo, chr21:43,193,374-43,198,244 y chr21:43,411,411-43,413,231) se asocian con un menor riesgo de aparición de cardiopatías congénitas que las de duplicación u otras variantes polimórficas (Single Nucleotide Variation, SNV) de genes como el CRELD1 y el GATA4, que predisponen a un mayor riesgo de padecerlas $(25,26)$.

La discapacidad intelectual en los niños con síndrome de Down no está determinada únicamente por el efecto del número de copias de algunos genes como el DYRK1A, pues también se ha observado que algunas variantes polimórficas de nucleótido único (SNV) influyen negativamente en su capacidad intelectual $(27,28)$. 
En Perú, y en muchos países latinoamericanos, el diagnóstico prenatal de aneuploidías no está protocolizado, y la legislación prohíbe la terminación de la gestación en el caso de anomalías congénitas (29). En tal sentido, en un reporte previo se observó una incidencia de neonatos con síndrome de Down hasta tres veces mayor que lo reportado mundialmente (30).

En este contexto, es importante determinar con mayor precisión cuáles son las variantes genéticas que podrían modificar negativamente el fenotipo de las aneuplodías (por ejemplo, de la trisomía 21), con el fin de conocer con exactitud el pronóstico y ofrecer tratamientos específicos.

\section{Materiales y métodos}

El estudio fue descriptivo y transversal. Se preservó la privacidad y la confidencialidad de los datos clínicos y genómicos de los sujetos de investigación y sus respectivas familias, siguiendo las directrices de las buenas prácticas clínicas y de ética de la investigación biomédica. El estudio fue autorizado por el Comité de Ética Independiente del Instituto Nacional de Salud del Niño-Breña y se contó con la firma del consentimiento informado por parte de los padres.

Durante el periodo de estudio, nacieron en los hospitales Regional y Antonio Lorena de Cusco 19 niños con aneuploidías, 16 de ellos con trisomía 21 libre y tres con trisomía 18; no se registró ningún paciente con trisomía 13.

Se utilizó un cuestionario estructurado para la recopilación de la información clínica y molecular, y se codificaron y encriptaron los datos de identificación de los pacientes. El análisis cromosómico por micromatrices (Chromosomal Microarray Analysis, CMA) se hizo en una muestra de sangre periférica, de la cual se extrajo el ADN genómico (250 ng). El ADN fue amplificado, etiquetado e hibridado, usando el protocolo GeneChip CytoScan $750 \mathrm{~K}$ Array $^{\mathrm{TM}}$ (Affymetrix, USA) y siguiendo las instrucciones del fabricante.

La prueba incluye 550.000 marcadores no polimorfos y 200.436 marcadores de polimorfismo de nucleótido único (Single Nucleotide Polymorphism, SNP). Las celdas en filas se escanearon y luego se analizaron mediante el programa informático Chromosome Analysis Suite (ChAS) ${ }^{\mathrm{TM}}$ (Affymetrix, USA). Las ganancias o pérdidas se consideraron si comprometían, por lo menos, 50 y 25 marcadores, respectivamente, y las regiones de homocigosidad, si abarcaban una longitud de por lo menos $5 \mathrm{Mb}$. Para determinar el número de recién nacidos vivos con aneuploidías autosómicas en los que se realizó el análisis cromosómico por micromatrices, se calculó el tamaño de la muestra según la fórmula $\left(n=\left[N^{*}\left(z^{2 *} p^{*} q\right)\right] /\left[e^{2 *}(N-1)+z^{2 *} p * q\right)\right.$, en la cual $N=7.413, z=99 \%$, $\mathrm{e}=5 \%$ y $\mathrm{p}$ correspondía a la incidencia de las aneuploidías 21 y 18 , por lo que se determinó que $n_{1}=5$ correspondía a la trisomía 21 y $n_{2}=2$ a la trisomía 18 . No se hizo el análisis cromosómico por micromatrices ni ningún estudio citogenético a los padres ni a la población en general.

Las variaciones en el número de copias se compararon con las bases de datos genómicos DECIPHER, y la de la University of California, Santa Cruz (UCSC), y se clasificaron en patogénicas, probablemente patogénicas, de significado incierto, probablemente benignas o benignas, según las recomendaciones del American College of Medical Genetics and Genomic (ACMG) $(31,32)$.

Los resultados del análisis cromosómico por micromatrices se clasificaron como anormales si se demostraban variaciones en el número de copias 
patogénicas o probablemente patogénicas, o si se presentaba, por lo menos, una región con una región de homocigosidad mayor de $10 \mathrm{Mpb}$ o si el total de las regiones de homocigosidad en las regiones autosómicas era mayor de 0,5\% (33).

Por último, se determinó la frecuencia de los resultados anormales del análisis cromosómico por micromatrices y se comparó con los estudios previos publicados. El análisis descriptivo se hizo mediante el uso de frecuencias y porcentajes.

\section{Resultados}

Entre diciembre del 2017 y noviembre del 2018, hubo en el Hospital Antonio Lorena 3.195 recién nacidos vivos y, en el Hospital Regional de Cusco, 4.218 ( $\mathrm{N}=7.413)$. El número de estos con síndrome de Down en las dos instituciones fue de 16, estimándose una incidencia de 2,16 por cada 1.000 recién nacidos vivos. Los antecedentes prenatales, el tipo de parto, las anomalías congénitas asociadas, las características antropométricas, la edad de los padres y la edad gestacional, se detallan en el cuadro 1. Tres de los recién nacidos presentaban síndrome de Edwards, es decir, una incidencia de 0,4 por cada 1.000 recién nacidos vivos. No se registró ningún paciente con síndrome de Patau.

La mediana del porcentaje de las regiones de homocigosidad fue de $0,47 \%$, con valores que fluctuaron entre 0,37 y $0,93 \%$. Tres de los neonatos tenían una región de homocigosidad mayor de $0,5 \%$ y ninguno presentó una región de homocigosidad en un cromosoma mayor de $10 \mathrm{Mb}$ que indicara una posible disomía uniparental (cuadro 2).

\begin{tabular}{|c|c|c|}
\hline \multicolumn{3}{|c|}{ Recién nacidos con trisomía 21 ( $\mathrm{n}=16)$} \\
\hline Sexo & $\mathbf{n}$ & $\%$ \\
\hline Masculino & 8 & 50 \\
\hline Femenino & 8 & 50 \\
\hline Antecedentes prenatales & $\mathbf{n}$ & $\%$ \\
\hline No & 5 & 31,25 \\
\hline Consumo de alcohol & 3 & 18,75 \\
\hline Fiebre en el primer trimestre & 2 & 12,5 \\
\hline Amenaza de aborto & 2 & 12,5 \\
\hline Amenaza de parto prematuro & 2 & 12,5 \\
\hline Parto & $\mathbf{n}$ & $\%$ \\
\hline Vaginal & 7 & 43,75 \\
\hline Cesárea & 9 & 56,25 \\
\hline Otras anomalías asociadas & $\mathbf{n}$ & $\%$ \\
\hline Malformación anorrectal & 2 & 12,5 \\
\hline Atresia esofágica & 1 & 6,25 \\
\hline Malformación cardíaca & 7 & 43,75 \\
\hline Antropometría al nacer & Mediana & Rango intercuartílico \\
\hline Peso (g) & 3.040 & $1.890-3.310$ \\
\hline Talla (cm) & 47.7 & $43-48$ \\
\hline Perímetro cefálico $(\mathrm{cm})$ & 33 & $28-35$ \\
\hline Edad parental & Mediana & Rango intercuartílico \\
\hline Padre & 32 & $20-46$ \\
\hline Madre & 29 & $19-42$ \\
\hline $\begin{array}{l}\text { Edad gestacional } \\
\text { Total }\end{array}$ & $\begin{array}{l}\text { Mediana } \\
\quad 38\end{array}$ & $\begin{array}{c}\text { Rango intercuartílico } \\
31-39\end{array}$ \\
\hline
\end{tabular}


Cuadro 2. Tamaño de las regiones de homocigosidad y coeficiente de endogamia en los recién nacidos vivos con aneuploidiías en Cusco

\begin{tabular}{|c|c|c|c|c|c|c|c|c|}
\hline Parentesco & $\begin{array}{l}\text { Grado de } \\
\text { relación }\end{array}$ & $\begin{array}{l}\text { Coeficiente de } \\
\text { endogamia }\end{array}$ & $\begin{array}{c}\text { Proporción teórica } \\
\text { idéntica en descendientes }\end{array}$ & $\begin{array}{c}\text { Rango de } \\
\text { Mb }\end{array}$ & $\begin{array}{l}\text { Promedio } \\
\text { Mb }\end{array}$ & Ranc & $\begin{array}{l}\text { go de } \\
\%\end{array}$ & $\begin{array}{c}\text { Número de } \\
\text { RNV con ROH }\end{array}$ \\
\hline Padres-hijos; hermanos & Primer & $1 / 4$ & $25 \%$ & 5401.080 & 720 & 18,7 & 37,5 & 0 \\
\hline Tío-sobrino; primos de primer grado doble & Segundo & $1 / 8$ & $12,50 \%$ & 270539 & 360 & 9,37 & 18,7 & 0 \\
\hline Primos de primer grado & Tercer & $1 / 16$ & $6,25 \%$ & 135269 & 180 & 4,69 & 9,34 & 0 \\
\hline Primos de segundo grado & Cuarto & $1 / 32$ & $3,13 \%$ & 68134 & 90 & 2,36 & 4,65 & 0 \\
\hline Primos de tercer grado & Quinto & $1 / 64$ & $1,56 \%$ & 1467 & 43,75 & 0,5 & 2,35 & 3 \\
\hline
\end{tabular}

$\mathrm{RNV}$ : recién nacidos vivos; $\mathrm{ROH}$ : regions of homocigocity

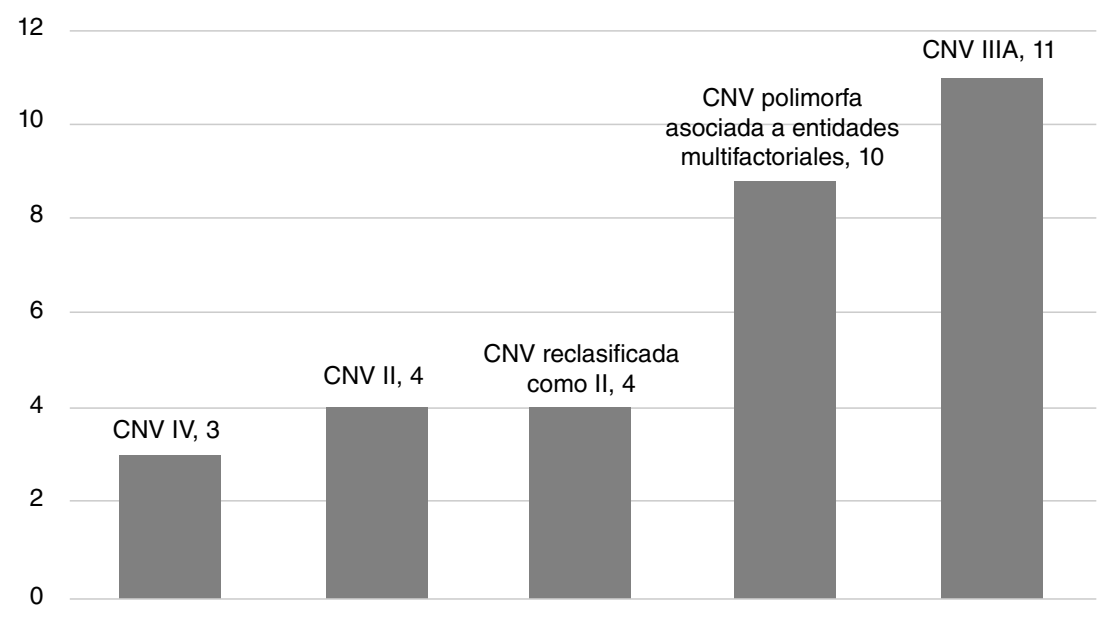

CNV: Copy number variation

Figura 1. Proporción de variantes en el número de copias encontradas en recién nacidos con trisomía 21 y trisomía 18. Las variantes en el número de copias de significado incierto (tipo IIIA) fueron las más frecuentes, seguidas por las polimorfas, las IIIA reclasificadas como probablemente patogénicas (II), las II y, por último, las benignas (IV).

En total, hubo 34 variaciones en el número de copias, con un tamaño variable que osciló entre 28 y $77.878 \mathrm{~kb}$ y una mediana de $165 \mathrm{~kb}$. En total, hubo 42 variaciones en el número de copias, con un tamaño variable que osciló entre 28 y $77.878 \mathrm{~kb}$ y una mediana de $165 \mathrm{~kb}$ entre variantes que van desde las patogénicas hasta las benignas, según la clasificación del ACMG. Las variantes patogénicas están relacionadas con la trisomía 18 y 21 . Se encontraron variaciones benignas en tres y probablemente benignas en un neonato. Hubo 10 variaciones polimorfas en el número de copias que se han asociado a carcinoma nasofaríngeo en cuatro, a fisura labio-palatina en tres, a obesidad precoz en dos y talla baja en uno. De las variaciones de capacidad patógena desconocida, 10 tuvieron un tamaño menor de $500 \mathrm{~kb}$. (figura 1).

En un recién nacido con trisomía 21, se encontraron otras tres variaciones en el número de copias probablemente patogénicas en 3q29, Xp22.33 y Xq28. Por otro lado, en una de las trisomías 18 , se encontró una variación probablemente patogénica en 16p12.2 (cuadro 3).

En dos neonatos, se hallaron, además, variaciones en el número de copias de ganancia mayores de $500 \mathrm{~kb}$ en los cromosomas 4 y 8 , clasificadas como de patogenia incierta, según DECIPHER y la USCC. Asimismo, se encontraron dos variaciones en el número de copias de pérdida que, según DECIPHER, incluían genes con un coeficiente de haploinsuficiencia menor o igual a $10 \%(Z Z Z 3, T T C 28)$ (cuadro 4). 
Cuadro 3. Variantes en el número de copias patogénicas y probablemente patogénicas en recién nacidos con trisomía 21 y trisomía 18

\begin{tabular}{|c|c|c|c|c|c|c|c|c|c|c|}
\hline Paciente & $\begin{array}{l}\text { Región } \\
\text { cromosómica }\end{array}$ & $\begin{array}{l}\text { Tipo de } \\
\text { CNV }\end{array}$ & Coordenadas & $\begin{array}{c}\text { Tamaño } \\
\text { (kb) }\end{array}$ & $\begin{array}{c}\text { Número } \\
\text { de } \\
\text { genes }\end{array}$ & Clase & $\begin{array}{c}\text { Genes } \\
\text { asociados } \\
\text { a fenotipo }\end{array}$ & $\begin{array}{l}\text { Dosis } \\
\text { génica+ }\end{array}$ & $\begin{array}{l}\text { Identificación } \\
\text { DECIPHER }\end{array}$ & $\begin{array}{l}\text { Fenotipo } \\
\text { esperado }\end{array}$ \\
\hline 1 & $21 q$ & Duplicación & $15,016,486 \_48,093,361$ & $>33.000$ & 511 & $\mathrm{I}$ & DYRK1A1 & 0 & & Síndrome de Down \\
\hline 2 & $21 q$ & Duplicación & $15,016,486 \_48,093,361$ & $>33.000$ & 511 & $\mathrm{I}$ & DYRK1A1 & 0 & & Síndrome de Down \\
\hline \multirow[t]{2}{*}{3} & $16 \mathrm{p} 12.2$ & Duplicación & 21,740,199_22,442,007 & 702 & & II & & & $\begin{array}{l}294453 \\
304664 ; \\
305863\end{array}$ & $\begin{array}{l}\text { Riñones en } \\
\text { herradura, } \\
\text { trombocitopenia, } \\
\text { TEA }\end{array}$ \\
\hline & $18 p 11.32 q 23$ & Duplicación & $136,227-78,013,728$ & $>77.000$ & 697 & 1 & & 0 & & \\
\hline 4 & $21 q$ & Duplicación & $15,016,486 \_48,093,361$ & $>33.000$ & 511 & 1 & DYRK1A1 & 0 & & Síndrome de Down \\
\hline 5 & $18 p 11.32 q 23$ & Duplicación & $136,227-78,013,728$ & $>77.000$ & 697 & 1 & & & & $\begin{array}{l}\text { Síndrome de } \\
\text { Edwards }\end{array}$ \\
\hline \multirow[t]{4}{*}{6} & $3 q 29$ & Deleción & $196,507,578-196,535,849$ & 28 & & II & PAK2 & $1 ; 16,97 \%$ & -- & Esquizofrenia, TEA \\
\hline & $21 q$ & Duplicación & $15,016,486-48,093,361$ & $>33.000$ & & I & DYRK1A1 & 0 & & Síndrome de Down \\
\hline & Xp22.33 & Duplicación & $535,572-644,544$ & 109 & & II & SHOX & 0 & -- & $\begin{array}{l}\text { Talla alta o baja y } \\
\text { TND }\end{array}$ \\
\hline & Xq28 & Duplicación & $152,927,530-153,102,573$ & 175 & 11 & II & $\begin{array}{l}\text { SLC6A8, } \\
\text { BCAP31 }\end{array}$ & 0 & -- & Hipoacusia \\
\hline 7 & $21 q$ & Duplicación & $15,016,486 \_48,093,361$ & $>33.000$ & 511 & I & DYRK1A1 & 0 & & Síndrome de Down \\
\hline
\end{tabular}

TEA: trastorno del espectro autista; CNV: copy number variation; TND: trastornos del neurodesarrollo

+ Según ClinGen y DECIPHER;

Cuadro 4. Variantes en el número de copias clasificadas como desconocidas que podrían ser reclasificadas como probablemente patogénicas

\begin{tabular}{|c|c|c|c|c|c|c|c|c|c|}
\hline Cromosoma & $\begin{array}{l}\text { Tamaño } \\
\text { (kb) }\end{array}$ & Nomenclatura de CMA & $\begin{array}{l}\text { Tipo de } \\
\text { CNV }\end{array}$ & $\begin{array}{l}\text { Número } \\
\text { de genes }\end{array}$ & $\begin{array}{l}\text { Genes } \\
\text { OMIM }\end{array}$ & $\begin{array}{c}\text { Haploinsuficiencia } \\
<10 \% \circ \\
\text { triplosensibilidad }\end{array}$ & Función & $\begin{array}{c}\text { Puntaje según } \\
\text { CNV Pathogenicity } \\
\text { Calculator }\end{array}$ & $\begin{array}{l}\text { Anomalías } \\
\text { asociadas }\end{array}$ \\
\hline \multirow[t]{5}{*}{$4 q 31.1$} & 691 & $\begin{array}{c}\text { arr[hg19] 4q31. } \\
\text { 1q31.21(140,937,627-141,628,406)x3 }\end{array}$ & Duplicación & 12 & UCP1 & -- & $\begin{array}{l}\text { Respiración } \\
\text { termogénica de las } \\
\text { mitocondrias }\end{array}$ & & $\begin{array}{l}\text { Comunicación } \\
\text { interauricular }\end{array}$ \\
\hline & & & & & MAML3 & -- & $\begin{array}{l}\text { Coactivador } \\
\text { transcripcional de } \\
\text { NOTCH }\end{array}$ & & \\
\hline & & & & & CLGN & -- & Espermatogénesis & & \\
\hline & & & & & ELMOD2 & -- & $\begin{array}{l}\text { Proteína activadora } \\
\text { de GTP }\end{array}$ & & \\
\hline & & & & & TBC1D9 & -- & $\begin{array}{l}\text { Actúa como } \\
\text { GTPasa activando } \\
\text { proteínas Rab }\end{array}$ & & \\
\hline \multirow[t]{5}{*}{$8 p 21.3$} & 1,285 & $\begin{array}{c}\operatorname{arr}[\text { hg19] 8p21.3(19,181,933- } \\
20,466,541) \times 3\end{array}$ & Duplicación & 14 & $L P L$ & -- & $\begin{array}{l}\text { Hidrólisis de } \\
\text { triglicéridos y VLDL }\end{array}$ & 0 & $\begin{array}{c}\text { No } \\
\text { especificado }\end{array}$ \\
\hline & & & & & LZST1 & -- & Crecimiento celular & & \\
\hline & & & & & SLC18A1 & -- & $\begin{array}{l}\text { Transportador } \\
\text { vesicular de } \\
\text { monoaminas }\end{array}$ & & \\
\hline & & & & & INTS10 & -- & $\begin{array}{l}\text { Subunidad } \\
\text { del complejo } \\
\text { integrador, } \\
\text { asociado a la } \\
\text { polimerasa ARN II }\end{array}$ & & \\
\hline & & & & & ATP6V1B2 & -- & $\begin{array}{l}\text { Acidificación de } \\
\text { compartimentos } \\
\text { intracelulares }\end{array}$ & & \\
\hline $1 \mathrm{p} 31.1$ & 108 & $\begin{array}{c}\operatorname{arr}[\text { hg19] } 1 \mathrm{p} 31.1(78,108,972- \\
78,216,987) \times 1\end{array}$ & Deleción & 3 & $Z Z 3$ & ZZZ3 & $\begin{array}{l}\text { Modificaciones } \\
\text { postraducción en } \\
\text { histonas }\end{array}$ & 0 & $\begin{array}{l}\text { Malformación } \\
\text { anorrectal }\end{array}$ \\
\hline $22 q 12.2$ & 324 & $\begin{array}{c}\text { arr[hg19] 22q12.1(28,751,664- } \\
29,076,146) \times 1\end{array}$ & Deleción & 3 & TTC28 & TTC28 & $\begin{array}{l}\text { Condensación de } \\
\text { los microtúbulos de } \\
\text { la zona media del } \\
\text { huso }\end{array}$ & 0.3 & $\begin{array}{l}\text { Malformación } \\
\text { anorrectal }\end{array}$ \\
\hline
\end{tabular}


No se pudo hacer el análisis cromosómico por micromatrices ni otros estudios citogenéticos a los padres de estos pacientes para tener una idea del estado de patogenia y evaluar si eran heredadas o de novo. En el mismo sentido, al no contar con este análisis en la población general, no fue posible determinar cómo las aneuploidias podrían influir en el riesgo de aparición de variaciones en el número de copias modificadoras del fenotipo o de su frecuencia poblacional.

\section{Discusión}

En un estudio previo en un hospital de Lima, se había estimado una incidencia del síndrome de Down de 5,7 por 1.000 recién nacidos vivos (30); esta cifra es mayor que la encontrada en el presente estudio (2,16 por 1.000), la cual corresponde a los casos de dos hospitales. Esta información debe confirmarse mediante vigilancia epidemiológica de todos los hospitales de la región y del país, para incorporarla de manera progresiva en el Estudio Colaborativo Latinoamericano de Malformaciones Congénitas (ECLAMC) y contar con una base de datos más exacta.

En un paciente con trisomía 21 se encontró una ganancia de $175 \mathrm{kpb}$ en el cromosoma X(q28), que comprometía nueve genes, entre ellos el BCAP31 y el SLC6A8, cuya duplicación se ha relacionado con hipoacusia no sindrómica (12). El gen BCAP31 (B Cell Receptor Associated Protein 31) codifica una proteína chaperona que se expresa en el retículo endoplasmático, cuya función es exportar proteínas secretadas desde dicho retículo y reconocer proteínas mal plegadas que están dirigidas hacia la vía de degradación asociada con este; además, sirve como receptor de carga para la exportación de proteínas transmembrana. Las variaciones en este gen se han relacionado con una alteración neurológica grave caracterizada por hipoacusia, distonía e hipomielinización central (MIM \# 300475) (34). Sin embargo, según ClinGen y DECIPHER, aún no se sabe si es un gen cuyo efecto depende del número de copias (gene dosage) presentes en el genoma.

En el gen SLC6A8, que es un transportador de creatina encargado de proveer de energía al músculo esquelético y cardíaco, se ha observado que las variaciones en el número de copias de ganancia están asociadas con hipoacusia (11). Este paciente se encontró una variación en el número de copias de ganancia en Xp22.38 que comprometía al gen SHOX, el cual es un factor de riesgo y de baja penetrancia relacionado con los trastornos del espectro autista y otros trastornos del neurodesarrollo como la discapacidad intelectual $(17,21)$.

Además, se ha observado una deleción de 28 kb en el cromosoma 3 (q29) que compromete parcialmente al gen PAK2 (p21, Prote ín-Activated Kinase2), el cual es una cinasa de serina o treonina que interviene en la supervivencia y el crecimiento celular por medio del citoesqueleto. El coeficiente de haploinsuficiencia de este gen es de 16,29\%, según DECIPHER, y de 1, según ClinGen, y las deleciones en él se han asociado con trastornos del espectro autista y esquizofrenia $(13-16,18,19)$. En ratones, se ha observado que dichas deleciones están involucradas en el desarrollo cerebral y la patogenia de dichos trastornos (20). Es así que este recién nacido con trisomía 21 tiene un gran riesgo de presentar trastornos del espectro autista, esquizofrenia, hipoacusia o una mayor discapacidad intelectual.

En otro paciente con trisomía 18, se observó una variación de ganancia de $702 \mathrm{kpb}$ en el número de copias de 12 genes en el cromosoma 16 (p12.2), 
la cual, según DECIPHER, se ha reportada como probablemente patogénica en tres pacientes (identificados como 294453,304664 y 305863 ) y se ha asociado con trombocitopenia, trastornos del espectro autista, discapacidad intelectual y riñones en herradura $(23,24)$.

Por otra parte, hubo tres pacientes con trisomía 21 y variaciones adicionales en el número de copias mayores de 500 kb en $4 q 31.1$ y $8 p 21.2$, así como otras que involucraban genes con haploinsuficiencia menor de 10 (cuadro 4), las cuales podrían reclasificarse como probablemente patogénicas por el tamaño y el contenido génico. En ese sentido, el paciente con estas variaciones en el número de copias, presentaba una malformación anorrectal, y se ha descrito que las variaciones en el gen TTC28 están relacionadas con anomalías congénitas del tubo digestivo (22).

Por último, dos pacientes con trisomía 21 y uno con trisomía 18, cuyos padres no declararon consanguinidad, tenían una región de homocigosidad en los cromosomas autosómicos de entre 0,5 y 2,35 \%, que corresponde a un coeficiente de endogamia $(F)$ de 1/64, lo que indica un grado de relación parental de quinto grado. Esta consanguinidad podría ser un factor de riesgo de enfermedades recesivas autosómicas o, incluso, ser un factor etiológico de la aneuploidia (35).

Al no contar con estudios genéticos de los padres, como el análisis cromosómico por micromatrices, no fue posible establecer si las variaciones incidentales en el número de copias eran heredadas o de novo y, tampoco, reclasificar aquellas de significado incierto. Sin embargo, las variaciones patogénicas o probablemente patogénicas están relacionadas con otras condiciones y pueden incrementar la gravedad de algunos síntomas de los pacientes con aneuploidias.

Por lo tanto, el fenotipo variable que observamos en los pacientes con aneuploidias se debería a las diferentes variantes observables en el genoma en combinación y, aunque en menor proporción, al medio ambiente y los cambios epigenéticos (figura 2).

Las aneuploidias en los recién nacidos son el grupo de enfermedades genéticas más frecuentes, por lo que es de suma importancia conocer y reconocer cuáles son los factores genéticos o ambientales que modifican el fenotipo de las personas con aneuploidias como el síndrome de Down, para poder advertir oportunamente las complicaciones o el pronóstico y ofrecerles una atención adecuada.

Algunos de los recién nacidos en esta cohorte pequeña, tenían otras variaciones en el número de copias, las cuales modificarían el fenotipo de forma negativa y los haría más propensos a sufrir o que se agraven algunas de las características clínicas propias de las aneuploidías, por ejemplo, la hipoacusia en el síndrome de Down, o a padecer otras comorbilidades no descritas o muy poco frecuentes, como la esquizofrenia, la talla alta o baja y los trastornos del espectro autista, sobre todo aquellos con trisomía 21. Muchas veces, el fenotipo observado en estos pacientes es producto,o no solo de la aneuploidía observada per se, sino de otras variantes genéticas (variaciones en el número de copias o las de nucleótido único), las cuales deberían explorarse con más precisión y a mayor escala. 


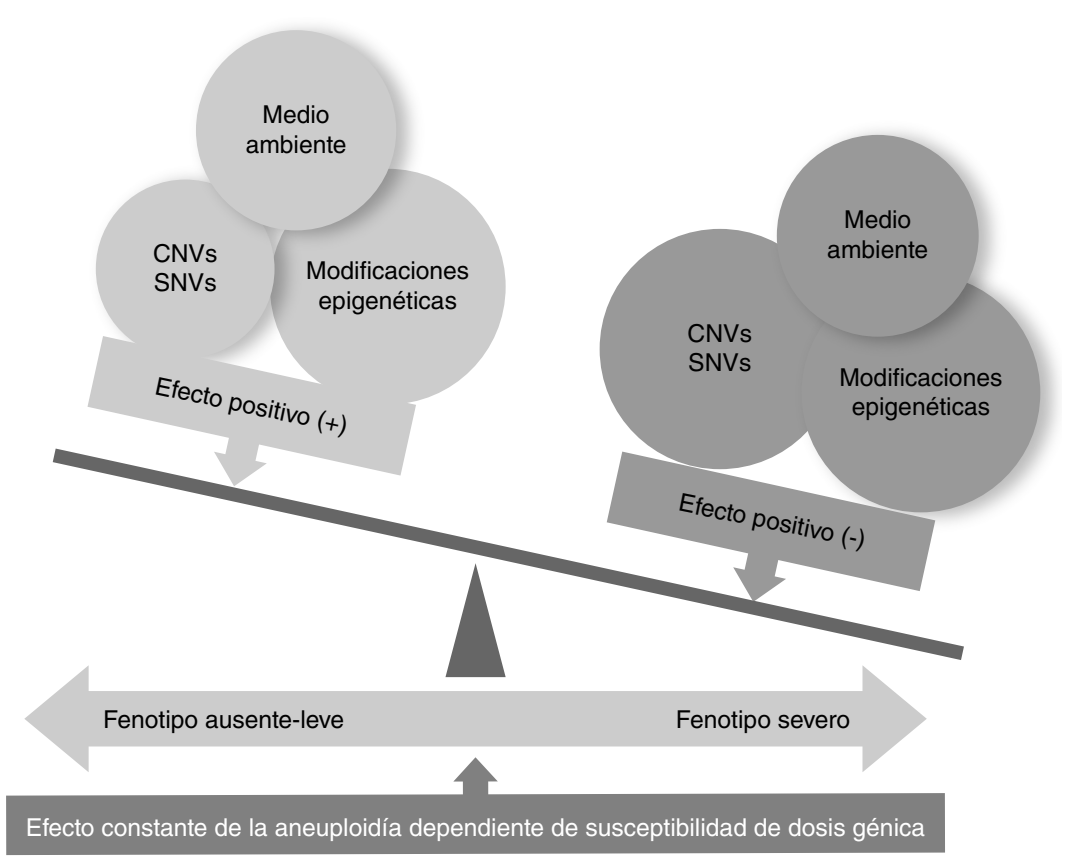

Figura 2. Interrelación de las variantes en el genoma de los niños con aneuploidías y el medio ambiente en la expresión final del fenotipo. Teóricamente las aneuploidías autosómicas provocan un fenotipo de manera constante en todos los pacientes (azul). Sin embargo, variantes genéticas diferentes a las aneuploidias ( o ) [variaciones en el número de copias (CNV) o las de nucleótido único (SNV)], conjuntamente con las modificaciones epigenéticas y factores del medio ambiente (por ejemplo, estimulación temprana deficiente) provocan un efecto negativo (rojo) en el fenotipo final (por ejemplo, mayor discapacidad intelectual, aparición de otros trastornos del neurodesarrollo).

\section{Agradecimientos}

A los padres de los pacientes que permitieron la recolección de los datos personales, y a las doctoras Anahí Venero Núñez y Carmen Ayuso García, por su contribución valiosa en la redacción del manuscrito.

\section{Referencias}

1. Hutaff-Lee C, Cordeiro L, Tartaglia N. Cognitive and medical features of chromosomal aneuploidy. Handb Clin Neurol. 2013;111:273-9.

https://doi.org/10.1016/B978-0-444-52891-9.00030-0

2. Nielsen J, Holm V, Haahr J. Prevalence of Edwards' syndrome. Clustering and seasonal variation? Humangenetik. 1975;26:113-6. https://doi.org/10.1007/BF00278437

3. Savva GM, Walker K, Morris JK. The maternal age-specific live birth prevalence of trisomies 13 and 18 compared to trisomy 21 (Down syndrome). Prenat Diagn. 2010;30:57-64. https://doi.org/10.1002/pd.2403

4. Sherman SL, Allen EG, Bean LH, Freeman SB. Epidemiology of Down syndrome. Ment Retard Dev Disabil Res Rev. 2007;13:221-7. https://doi.org/10.1002/mrdd.20157

5. Abarca-Barriga HH, Chávez-Pastor MA, Trubnykova M, Vásquez F, Poterico JA. Chromosomal microarray analysis in Peruvian children with delayed psychomotor development or intellectual disability. Rev Peru Med Exp Salud Pública. 2017;34:572-4. https://doi.org/10.17843/rpmesp.2017.343.2741

6. de Smith AJ, Trewick AL, Blakemore AIF. Implications of copy number variation in people with chromosomal abnormalities: Potential for greater variation in copy number state may contribute to variability of phenotype. Hugo J. 2010;4:1-9. https://doi.org/10.1007/s11568-010-9144-z

7. Stranger BE, Forrest MS, Dunning M, Ingle CE, Beazley C, Thorne N, et al. Relative impact of nucleotide and copy number variation on gene expression phenotypes. Science. 2007;315:848-53. https://doi.org/10.1126/science.1136678 
8. Henrichsen CN, Vinckenbosch N, Zöllner S, Chaignat E, Pradervand S, Schütz F, et al. Segmental copy number variation shapes tissue transcriptomes. Nat Genet. 2009;41:424-9. https://doi.org/10.1038/ng.345

9. Girirajan S, Eichler EE. Phenotypic variability and genetic susceptibility to genomic disorders. Hum Mol Genet. 2010;19:R176-87. https://doi.org/10.1093/hmg/ddq366

10. Rosenfeld JA, Coe BP, Eichler EE, Cuckle H, Shaffer LG. Estimates of penetrance for recurrent pathogenic copy-number variations. Genet Med. 2013;15:478-81. https://doi.org/10.1038/gim.2012.164

11. Schiaffino MC, Bellini C, Costabello L, Caruso U, Jakobs C, Salomons GS, et al. X-linked creatine transporter deficiency: Clinical description of a patient with a novel SLC6A8 gene mutation. Neurogenetics. 2005;6:165-8. https://doi.org/10.1007/s10048-005-0002-4

12. Rosenberg C, Freitas ÉL, Uehara DT, Auricchio MTBM, Costa SS, Oiticica J, et al. Genomic copy number alterations in non-syndromic hearing loss. Clin Genet. 2016;89:473-7. https://doi.org/10.1111/cge.12683

13. Willatt L, Cox J, Barber J, Cabanas ED, Collins A, Donnai D, et al. 3q29 microdeletion syndrome: Clinical and molecular characterization of a new syndrome. Am J Hum Genet. 2005;77:154-60. https://doi.org/10.1086/431653

14. Quintero-Rivera F, Sharifi-Hannauer P, Martinez-Agosto JA. Autistic and psychiatric findings associated with the 3q29 microdeletion syndrome: Case report and review. Am J Med Genet A. 2010;152A:2459-67. https://doi.org/10.1002/ajmg.a.33573

15. Carroll LS, Williams HJ, Walters J, Kirov G, O'Donovan MC, Owen MJ. Mutation screening of the 3q29 microdeletion syndrome candidate genes DLG1 and PAK2 in schizophrenia. Am J Med Genet Part B Neuropsychiatr Genet. 2011;156B:844-9. https://doi.org/10.1002/ajmg.b.31231

16. Cooper GM, Coe BP, Girirajan S, Rosenfeld JA, Vu T, Baker C, et al. A copy number variation morbidity map of developmental delay. Nat Genet. 2011;43:838-46.

https://doi.org/10.1038/ng.909

17. Iossifov I, O'Roak BJ, Sanders SJ, Ronemus M, Krumm N, Levy D, et al. The contribution of de novo coding mutations to autism spectrum disorder. Nature. 2014;515:216-21. https://doi.org/10.1038/nature13908

18. Warnica W, Merico D, Costain G, Alfred SE, Wei J, Marshall CR, et al. Copy number variable microRNAs in schizophrenia and their neurodevelopmental gene targets. Biol Psychiatry. 2015;77:158-66. https://doi.org/10.1016/..biopsych.2014.05.011

19. Zeng Y, Broxmeyer HE, Staser K, Chitteti BR, Park S-J, Hahn S, et al. Pak2 regulates hematopoietic progenitor cell proliferation, survival, and differentiation. Stem Cells Dayt Ohio. 2015;33:1630-41. https://doi.org/10.1002/stem.1951

20. Wang Y, Zeng C, Li J, Zhou Z, Ju X, Xia S, et al. PAK2 haploinsufficiency results in synaptic cytoskeleton impairment and autism-related behavior. Cell Rep. 2018;24:2029-41. https://doi.org/10.1016/j.celrep.2018.07.061

21. Singh H, Tiwari P, Bhavi V, Chaudhary PS, Suravajhala P, Mohan MK, et al. Application of chromosomal microarray for evaluation of idiopathic short stature in Asian Indian children: A pilot study. Indian J Endocrinol Metab. 2018;22:100-6. https://doi.org/10.4103/ijem.IJEM 202_17

22. Meier N, Bruder E, Lapaire O, Hoesli I, Kang A, Hench J, et al. Exome sequencing of fetal anomaly syndromes: Novel phenotype-genotype discoveries. Eur J Hum Genet. 2019;27:730-7. https://doi.org/10.1038/s41431-018-0324-y

23. DECIPHER v9.7: Mapping the clinical genome. Fecha de consulta: 20 mayo de 2016. Disponible en: https://decipher.sanger.ac.uk/browser

24. Firth HV, Richards SM, Bevan AP, Clayton S, Corpas M, Rajan D, et al. DECIPHER: Database of Chromosomal Imbalance and Phenotype in Humans Using Ensembl Resources. Am J Hum Genet. 2009;84:524-33. https://doi.org/10.1016/j.ajhg.2009.03.010

25. Sailani MR, Makrythanasis P, Valsesia A, Santoni FA, Deutsch S, Popadin K, et al. The complex SNP and CNV genetic architecture of the increased risk of congenital heart defects in Down syndrome. Genome Res. 2013;23:1410-21. https://doi.org/10.1101/gr.147991.112

26. Rambo-Martin BL, Mulle JG, Cutler DJ, Bean LJH, Rosser TC, Dooley KJ, et al. Analysis of copy number variants on chromosome 21 in Down syndrome-associated congenital heart defects. G3 (Bethesda). 2017;8:105-11. https://doi.org/10.1534/g3.117.300366 
27. Mégarbané A, Noguier F, Stora S, Manchon L, Mircher C, Bruno R, et al. The intellectual disability of trisomy 21: Differences in gene expression in a case series of patients with lower and higher IQ. Eur J Hum Genet. 2013;21:1253-9. https://doi.org/10.1038/ejhg.2013.24

28. Park J, Chung KC. New perspectives of Dyrk1A role in neurogenesis and neuropathologic features of Down syndrome. Exp Neurobiol. 2013;22:244-8. https://doi.org/10.5607/en.2013.22.4.244

29. Rodríguez-Cadilla MR. Consideraciones bioéticas y jurídicas de la información genética y el diagnóstico prenatal. Vox Juris. 2015;28:15-40.

30. Abarca-Barriga H. Perfil epidemiológico de las anomalías genéticas y congénitas en el Servicio de Citogenética y Citopatología del Hospital Nacional Guillermo Almenara Irigoyen (tesis). Lima: Universidad Nacional Mayor de San Marcos; 2007.

31. Carreira IM, Ferreira SI, Matoso E, Pires LM, Ferrão J, Jardim A, et al. Copy number variants prioritization after array-CGH analysis - a cohort of 1000 patients. Mol Cytogenet. 2015;8:103-11. https://doi.org/10.1186/s13039-015-0202-z

32. Riggs ER, Andersen EF, Cherry AM, Kantarci S, Kearney H, Patel A, et al. Technical standards for the interpretation and reporting of constitutional copy-number variants: A joint consensus recommendation of the American College of Medical Genetics and Genomics (ACMG) and the Clinical Genome Resource (ClinGen). Genet Med. 2019;22:245-57. https://doi.org/10.1038/s41436-019-0686-8

33. Fan Y-S, Ouyang X, Peng J, Sacharow S, Tekin M, Barbouth D, et al. Frequent detection of parental consanguinity in children with developmental disorders by a combined $\mathrm{CGH}$ and SNP microarray. Mol Cytogenet. 2013;6:38-43. https://doi.org/10.1186/1755-8166-6-38

34. Rinaldi B, van Hoof E, Corveleyn A, van Cauter A, de Ravel T. BCAP31-related syndrome: The first de novo report. Eur J Med Genet. 2019;103732. https://doi.org/10.1016/j.ejmg.2019.103732

35. Sayee R, Thomas IM. Consanguinity, non-disjunction, parental age and Down's syndrome. J Indian Med Assoc. 1998;96:335-7. 